\title{
Glossing conventions
}

\begin{tabular}{|c|c|c|c|}
\hline 1 & first person & OPT & optative \\
\hline 2 & second person & PART & partitive \\
\hline 3 & third person & PERS & Personal \\
\hline AN & animate & PL & plural \\
\hline APPL & applicative & PRO & pronoun \\
\hline APPR & apprehensive & PROX & proximal \\
\hline ART & article & PSSR & possessor \\
\hline ASSOC & associative & PST & past \\
\hline ATTRIB & attributive & PURP & purposive \\
\hline AUG & augmentative & RD & reduplicant \\
\hline CAP & capability & REAL & realis \\
\hline CAUS & causative & REL & relativiser \\
\hline $\mathrm{CF}$ & counterfactual & REP & repetitive \\
\hline CLI & Class I & $R / R$ & reciprocal/reflexive \\
\hline CLII & Class II & SBJ & subject \\
\hline COLL & collective & SEQ & sequential \\
\hline COM & comitative & SG & singular \\
\hline COMPL & completive & SPEC & specific \\
\hline COND & conditional & SUBR & subordinator \\
\hline CONST & construct morpheme & TR & transitive \\
\hline DEM1 & demonstrative 1 & & \\
\hline DEM2 & demonstrative 2 & & \\
\hline DER & derivational morpheme & & \\
\hline DET & determiner & & \\
\hline DETR & detransitiviser & & \\
\hline DIM & diminutive & & \\
\hline DU & dual & & \\
\hline DIST & distal & & \\
\hline EMPH & emphatic & & \\
\hline EXCL & exclusive & & \\
\hline HORT & hortative & & \\
\hline HUM & human & & \\
\hline INAN & inanimate & & \\
\hline INCL & inclusive & & \\
\hline INTS & intensifier & & \\
\hline IPFV & imperfective & & \\
\hline IRR & irrealis & & \\
\hline LOC & locative & & \\
\hline NEG & negative & & \\
\hline MED & medial & & \\
\hline NHUM & non-human & & \\
\hline NSPEC & non-specific & & \\
\hline OBJ & object & & \\
\hline $\mathrm{OBL}$ & oblique & & \\
\hline ORD & ordinal & & \\
\hline
\end{tabular}


